\title{
Positional Connected Component Labeling Algorithm
}

\author{
G. Gayathri Devi* and C. P. Sumathi \\ Department of Computer Science, SDNB Vaishnav College for Women, \\ Tamil Nadu, India; mail2gg@yahoo.co.in; drcpsumathi@gmail.com
}

\begin{abstract}
This paper presents a new positional connected component labeling algorithm to label the connected component and to give the number of components present in the binary image. The algorithm is based on the position value of the foreground pixel of the image. All the connected components are identified by the algorithm and are assigned by a new label or the same label of their adjacent component. Experiments are conducted by applying proper threshold technique on various images from the datasets collected and tagged by the ICDAR Robust Reading Dataset Collection Team. It is observed that the proposed method finds the entire connected components in the image.
\end{abstract}

Keyword: Connected Component, Labeling, Object Extraction

\section{Introduction}

Connected Component Labeling (CCL) in an image is an important operation in pattern recognition systems. Many CCL algorithms have been proposed by the researchers as there are plenty of useful applications in pattern recognition like document analysis, detection of vehicle license plate, keyword based image search, identification of parts in industrial automation, face identification, content based retrieval, medical image analysis, video processing, page segmentation, etc. The algorithm assigns unique labels to different, disjoint connected components. The most common labeling algorithms are Two Pass Algorithm, Multiple Scan Algorithm, Contour Tracing Algorithm, Parallel Processing Algorithm, Single Pass Algorithm etc. and these techniques have their benefits and drawbacks.

This paper is organized as follows: Section 2 briefs the related work carried out by the researchers, Section 3 explains the relationship of pixel, Section 4 discusses the newly proposed Positional Connected Component Labeling Algorithm, Section 5 explains the experimental results and Section 6 concludes the paper with future work.

\section{Related Work}

Fu Chang et al. ${ }^{1}$ proposed a method to find connected components from binary images using a contour tracing technique to detect component contours and also to fill in interior areas. The component points are traced by this algorithm by a single pass to assign a label. Yuhai et al. ${ }^{2}$ projected a suitable connected component algorithm for hardware implementation or image processing system on Field-Programmable Gate Array (FPGA). This method is one-scan algorithm for labeling connected components in binary images based on sequentially processing two adjacent rows.

Phaisarn et al. ${ }^{3}$ proposed pixel-based scan mask and the block-based scan mask scan masks for connected components labeling in binary image. The block-based scan mask is, transformed to a near-optimal decision tree to reduce the computation time. Mehdi et al. ${ }^{4}$ provided a parallel version of Suzuki's sequential connected component algorithm in order to speed up the labeling process.

An improved single pass algorithm was proposed by $\mathrm{Ni} \mathrm{Ma}$ et al. ${ }^{5}$ which reduce the memory. The algorithm reassigns and reuses labels of each row to minimize the size of both the equivalence and region data tables.

${ }^{*}$ Author for correspondence 
The approach used by Rakhmadi et al. ${ }^{6}$ was a loop back connected component labeling algorithm that helped the system to distinguish the object detected according to their label. This technique reduced the searching time for locating the object by focusing only on the suspected object based on certain features defined.

David et al. ${ }^{7}$ used an efficient technique for distributing and coalescing data as well as efficient combinations of task and data parallelism. Their connected components algorithm performed parallel merging that limited the updating during iterative steps. Tetsuo et al. ${ }^{8}$ presented two in-place algorithms, one for counting the number of connected components and the other for labeling the connected components. Both of them run in linear time and need no extra array other than an input array.

\section{Pixel Relationship}

The pixels of a binary image are 0's and 1's. 1's will be used to denote foreground pixels and the 0's background pixels. In image processing not only the value of a particular pixel is important, but also the values of its neighbors are used when processing that pixel. A pixel at coordinate $(\mathrm{P}(\mathrm{x}$, $y)$ ) has four direct neighbors $\mathrm{N}_{4}(\mathrm{P})$ and four diagonal neighbors. $\mathrm{N}_{4}(\mathrm{P})$ of $\mathrm{P}(\mathrm{x}, \mathrm{y})$ includes $\mathrm{P}(\mathrm{x}-1, \mathrm{y}), \mathrm{P}(\mathrm{x}+1, \mathrm{y})$, $\mathrm{P}(\mathrm{x}, \mathrm{y}-1)$ and $\mathrm{P}(\mathrm{x}, \mathrm{y}+1)$ referred as West, East, North and South (Figure 1a) respectively. $N_{D}(P)$ of $P(x, y)$ includes $\mathrm{P}(\mathrm{x}-1, \mathrm{y}-1), \mathrm{P}(\mathrm{x}+1, \mathrm{y}-1), \mathrm{P}(\mathrm{x}-1, \mathrm{y}+1)$ and $\mathrm{P}(\mathrm{x}+1, \mathrm{y}+1)$ referred as Northwest, Northeast, Southwest, Southeast (Figure $1 \mathrm{~b}$ ) respectively. The $\mathrm{N}_{4}(\mathrm{P})$ and $\mathrm{N}_{\mathrm{D}}(\mathrm{P})$ are together known as 8-neighbors of the pixel $\mathrm{P}$, denoted by $\mathrm{N}_{8}(\mathrm{P})$.

Three types of connectivity can be defined between a pixel $(\mathrm{P})$ and a pixel $(\mathrm{Q})$. They are

i) 4-connectivity - connected if $\mathrm{q}$ is in $\mathrm{N}_{4}(\mathrm{P})$

ii) 8-connectivity - connected if $\mathrm{q}$ is in $\mathrm{N}_{8}(\mathrm{P})$

iii) $\mathrm{m}$-connectivity - connected if $\mathrm{q}$ is in $\mathrm{N}_{4}(\mathrm{P})$ or if $\mathrm{q}$ is in $\mathrm{N}_{\mathrm{D}}(\mathrm{P})$ and $\mathrm{N}_{4}(\mathrm{P}) \cap \mathrm{N}_{4}(\mathrm{Q})=\varnothing$

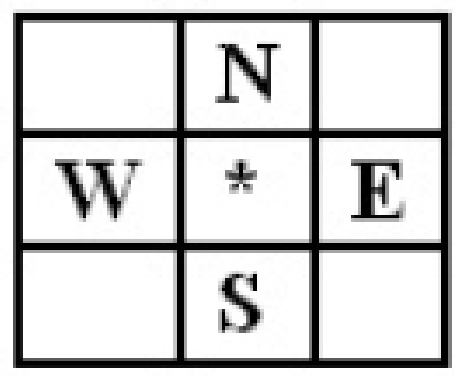

Figure 1a. Four neighborhood $\mathrm{N}_{4}$.
A pixel $P \times S$ is said to be connected to $Q \times S$ if there is a path from $P$ to $Q$ consisting entirely of pixels of $S$.

\section{Proposed Work}

A set of pixels in which each pixel is connected to all other pixels is called a connected Component. A component labeling algorithm finds all connected components in an image and assigns a unique label to all points in the same component. The proposed Positional Connected Component Labeling (PCCL) algorithm is based on 8-connectivity to find all connected components in an image, assigns an unique label to all points in the same component and find number of components present in the image. The algorithm for PCCL is based on the position of the white pixels in the image and is formulated as follows:

\subsection{Positional Connected Component Labeling (PCCL) Algorithm}

Input: Binary Image Matrix (I)

Output: Connected Component Labeled Matrix (L), Numbers_of_Components

Step 1: Find the foreground (white) pixel and record the foreground column position of the binary matrix image in a matrix (Position Matrix).

Step 2: Unmark all the cells of the Position Matrix(PM).

Step 3: Find the minimum position value (MinPos) and maximum position value (MaxPos) from the Position Matrix.

Step 4: Set the value of LABEL to 0.Current Row (CR) to 1.AV []$=\{\}, P V[]=\{\}$

Step 5: Get the first unmarked value (umv) from the Position Matrix (PV [] = umv). Set CR to the Row Number of umv. FLAG = NOTPREVMARKED. If no unmarked cell found, then go to step 16 .

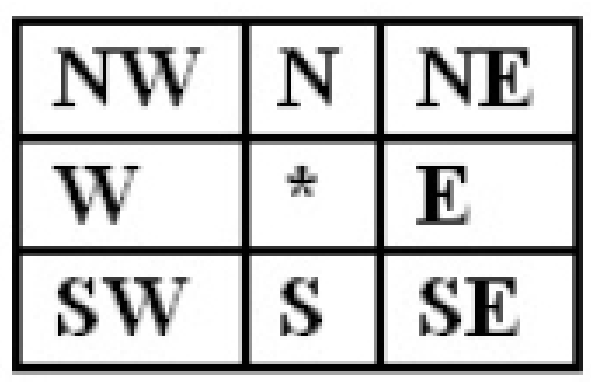

Figure 1b. Eight Neighborhood $\mathrm{N}_{8}$. 
Step 6: Find out the adjacent value (AV []) of the position values (PV []) from $\mathrm{PM}$. The adjacent values are $\mathrm{P}-1, \mathrm{P}$ and $\mathrm{P}+1$ for a value $\mathrm{P}$. If $\mathrm{P}$ is to be MinPos, then the adjacent values are $\mathrm{P}$ and $\mathrm{P}+1$. If $\mathrm{P}$ is to be MaxPos, then the adjacent values are $\mathrm{P}-1$ and $\mathrm{P}$.

Step 7: Search for the AV [] in the CR, CR-1 and $\mathrm{CR}+1$. Mark the corresponding cell. If any of these cells are already marked, change the FLAG= PREVMARKED, LABEL= LABEL assigned to the already marked cell. (Do not include CR-1 for the first row and $\mathrm{CR}+1$ for the last row).

Step 8: Increment CR by one.

Step 9: Scan CR and find the adjacent values (AV []) of cells marked by step 7 in the row CR.

Step 10: PV [] = AV []. Go to step 6if AV [] = $\varnothing$ or CR > LAST ROW.

Step 12: Assign LABEL value for the corresponding cells marked during this pass in the Input Image (I).

Step 13: If FLAG IS NOTPREVMARKED then increment LABEL value by one.

Step 14: If any unmarked cells found go to step 5 .

Step 15: Number_of_Components= LABEL -1 .

Step 16: Stop the procedure.
To illustrate the proposed method, a small binary image Figure 2a is taken. The column position of white pixel in image is recorded in a matrix called position matrix is (Figure 2). The values of MinPos, Maxpos are 1 and 10 respectively known from Figure $2 b$.

\section{Initilization:}

Set Label=1, CR=1, MinPos=1, MaxPos=10.

Pass I:

Get the first unmarked value (Figure 3a) from the Position Matrix. The unmarked value is $1 . P V[]=\{1\}$, FLAG $=$ NOTPREVMARKED according to step 5.

Pass i: The adjacent values of 1 are 1 and $2 . \operatorname{AV}[]=\{1,2\}$. $\mathrm{CR}=1$.The $\mathrm{AV}$ values are searched in $\mathrm{CR}$ and $\mathrm{CR}+1$ and those cells (1, 2 in Row 1 and 2 in Row 2) are marked (Figure $3 b$ ). Now CR is incremented by 1 $(\mathrm{CR}=2)$. The cell marked in CR is $2(\mathrm{PV}[]=\{2\})$.

Pass ii: The Adjacent values of 2 are $\operatorname{AV}[]=\{1,2,3\}$. Find those values in Row 1,2 and 3 as $\mathrm{CR}=2$ and those cells ( 3 in Row 1 and 3 in Row 2) are marked (Figure 3c).CR is incremented by $1(\mathrm{CR}=3)$. There is no cell marked in Row $3(\mathrm{AV}[]=\varnothing)$ ).First Pass is Over. Assign Label $=1$ to the corresponding

\begin{tabular}{|c|c|c|c|c|c|c|c|c|c|}
\hline 1 & 1 & 1 & $\mathbf{0}$ & $\mathbf{0}$ & $\mathbf{0}$ & 1 & $\mathbf{O}$ & $\mathbf{0}$ & 1 \\
\hline $\mathbf{O}$ & 1 & 1 & $\mathbf{O}$ & $\mathbf{O}$ & 1 & $\mathbf{0}$ & 1 & $\mathbf{O}$ & 1 \\
\hline $\mathbf{O}$ & $\mathbf{O}$ & $\mathbf{0}$ & $\mathbf{0}$ & 1 & $\mathbf{0}$ & 1 & $\mathbf{0}$ & $\mathbf{O}$ & 1 \\
\hline $\mathbf{O}$ & $\mathbf{O}$ & $\mathbf{O}$ & 1 & $\mathbf{O}$ & 1 & $\mathbf{O}$ & 1 & 1 & $\mathbf{O}$ \\
\hline $\mathbf{O}$ & $\mathbf{O}$ & 1 & 1 & $\mathbf{0}$ & 1 & 1 & $\mathbf{O}$ & 1 & $\mathbf{O}$ \\
\hline 1 & 1 & o & $\mathbf{0}$ & 1 & 1 & $\mathbf{0}$ & 1 & $\mathbf{O}$ & 1 \\
\hline $\mathbf{0}$ & 1 & 1 & 1 & O & $\mathbf{O}$ & 1 & 1 & 1 & $\mathbf{O}$ \\
\hline
\end{tabular}

Figure 2a. Input Image.

\begin{tabular}{|c|c|c|c|c|c|}
\hline $\mathbf{1}$ & 2 & 3 & 7 & 10 & \\
\hline 2 & 3 & 6 & 8 & 10 & \\
\hline 5 & 7 & \multicolumn{3}{|c|}{10} & \\
\hline 4 & 6 & \multicolumn{2}{|l|}{8} & 9 & \\
\hline 3 & 4 & 6 & 7 & 9 & \\
\hline $\mathbf{1}$ & 2 & 5 & 6 & 9 & 10 \\
\hline 2 & 3 & 4 & 7 & 8 & 9 \\
\hline
\end{tabular}

Figure 2b. Position Matrix.
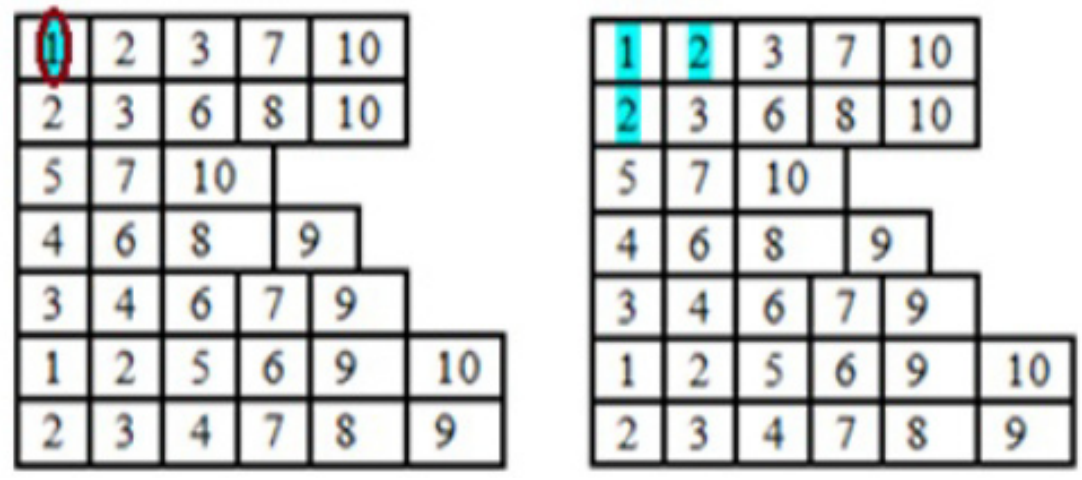

Figure 3a. First unmarked Cell in Pass - I. Figure 3b. Pass I - i.

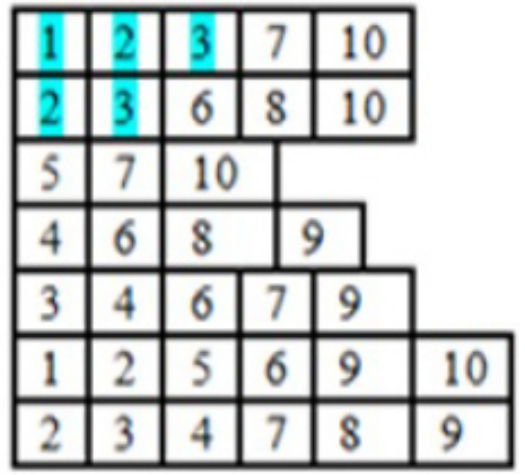

Figure 3c. Pass II - ii. 
marked cells in the Input Image (Figure 4). As FLAG is NOTPREVMARKED, LABEL is incremented by one. $(\mathrm{LABEL}=2)$

Pass II:

Get the first unmarked value from the PM (Figure 5a). The unmarked value is $1 . P V[]=\{7\}$. Set FLAG $=$ NOTPREVMARKED.

Pass i: The adjacent values of 7 are 6,7 and $8 . \mathrm{AV}[]=$ $\{6,7,8\}$. Now $C R=1$. The AV values are searched in CR and CR +1 and those cells $(6,7,8$ in Row 1 and 2 in Row 2) are marked (Figure 5b). Now CR is

\begin{tabular}{|l|l|l|l|l|l|l|l|l|l|}
\hline $\mathbf{1}$ & $\mathbf{1}$ & $\mathbf{1}$ & $\mathbf{0}$ & $\mathbf{0}$ & $\mathbf{0}$ & $\mathbf{1}$ & $\mathbf{0}$ & $\mathbf{0}$ & $\mathbf{1}$ \\
\hline $\mathbf{0}$ & $\mathbf{1}$ & $\mathbf{1}$ & $\mathbf{0}$ & $\mathbf{0}$ & $\mathbf{1}$ & $\mathbf{0}$ & $\mathbf{1}$ & $\mathbf{0}$ & $\mathbf{1}$ \\
\hline $\mathbf{0}$ & $\mathbf{0}$ & $\mathbf{0}$ & $\mathbf{0}$ & $\mathbf{1}$ & $\mathbf{0}$ & $\mathbf{1}$ & $\mathbf{0}$ & $\mathbf{0}$ & $\mathbf{1}$ \\
\hline $\mathbf{0}$ & $\mathbf{0}$ & $\mathbf{0}$ & $\mathbf{1}$ & $\mathbf{0}$ & $\mathbf{1}$ & $\mathbf{0}$ & $\mathbf{1}$ & $\mathbf{1}$ & $\mathbf{0}$ \\
\hline $\mathbf{0}$ & $\mathbf{0}$ & $\mathbf{1}$ & $\mathbf{1}$ & $\mathbf{0}$ & $\mathbf{1}$ & $\mathbf{1}$ & $\mathbf{0}$ & $\mathbf{1}$ & $\mathbf{0}$ \\
\hline $\mathbf{1}$ & $\mathbf{1}$ & $\mathbf{0}$ & $\mathbf{0}$ & $\mathbf{1}$ & $\mathbf{1}$ & $\mathbf{0}$ & $\mathbf{1}$ & $\mathbf{0}$ & $\mathbf{1}$ \\
\hline $\mathbf{0}$ & $\mathbf{1}$ & $\mathbf{1}$ & $\mathbf{1}$ & $\mathbf{0}$ & $\mathbf{0}$ & $\mathbf{1}$ & $\mathbf{1}$ & $\mathbf{1}$ & $\mathbf{0}$ \\
\hline
\end{tabular}

Figure 4. Image (I) after Pass I. incremented by $1(\mathrm{CR}=2)$. The cell marked in $\mathrm{CR}$ is 6 and $8(\mathrm{PV}[]=\{6,8\}$.

Pass ii: The Adjacent values of 6 and 8 are AV []$=\{5,6,7$, $8,9\}$. Find those values in Row 1,2 and 3 as $C R=2$ and those cells (5 and 7 in Row 3 ) are marked (Figure $5 \mathrm{c})$.CR is incremented by $1(\mathrm{CR}=3)$. The cell marked in CR are 5 and 7 (PV []$=\{5,7\}$.

Pass iii: The Adjacent values of 5 and 7 are AV []$=\{4,5,6$, $7,8\}$. Find those values in Row 2, 3 and 4 as $C R=3$ and those cells (4, 6 and 8 in Row 4$)$ are marked (Figure 6a).CR is incremented by $1(\mathrm{CR}=4)$. The cell marked in CR are 4, 6 and $8(\mathrm{PV}[]=\{4,6,8\}$.

Pass iv: The Adjacent values of 4, 6 and 8 are AV [] = $\{3,4,5,6,7,8,9\}$. Find those values in Row 3,4 and 5 as $\mathrm{CR}=4$ and those cells ( 9 in Row 4 and 3, 4, 6, 7 and 9 in Row 5) are marked (Figure 6b). $\mathrm{CR}$ is incremented by $1(\mathrm{CR}=5))$. The cell marked in CR are 4, 6 and $8(\mathrm{PV}[]=\{3,4,6,7,9\}$.

Pass v: The Adjacent values of 3, 4, 6, 7 and 9 are AV [] = $\{2,3,4,5,6,7,8,9,10\}$. Find those values in Row 4, 5 and 6 as $\mathrm{CR}=5$ and those cells $(2,5,6,9$ and 10 in Row 6) are marked (Figure 6c).CR is incremented by $1(\mathrm{CR}=6))$. The cell marked in CR are 2, 5, 6, 9 and $10(\mathrm{PV}[]=\{2,5,6,9,10\}$.

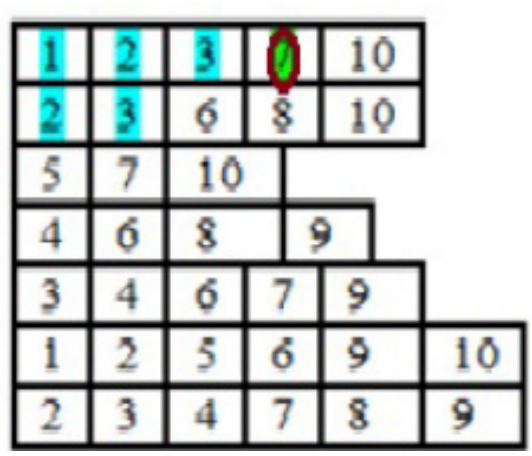

Figure 5a. First Unmarked Cell in PassII. Figure 5b. Pass II - i.

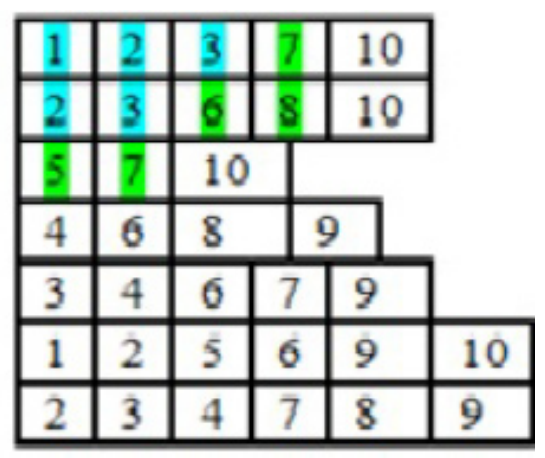

Figure 5c. Pass II - ii.

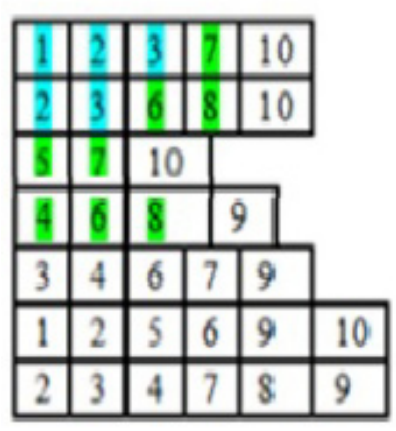

Figure 6a. Pass II-iii.

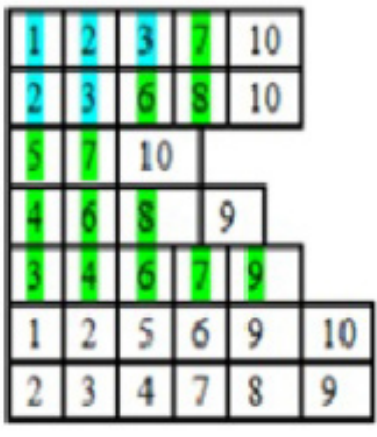

Figure 6b. Pass II-iv.

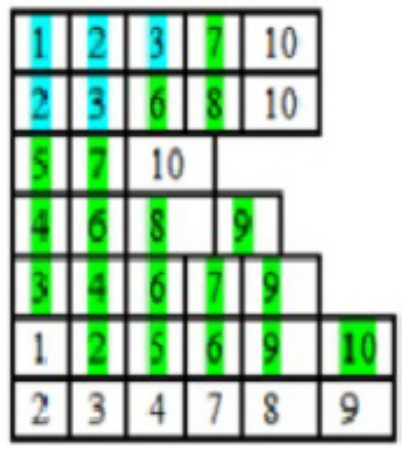

Figure 6c. Pass II-v.

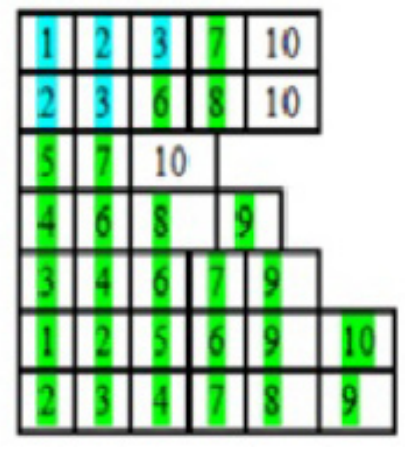

Figure 6d. Pass II -vi. 
Pass vi: The Adjacent values of 2, 5, 6, 9 and 10 are AV [] = $\{1,2,3,4,5,6,7,8,9,10\}$. Find those values in Row 5, 6 as CR $=6$ and those cells ( 1 in Row 5 and 2, 3, 4, 7, 8, 9 in Row 6) are marked (Figure 6d). $\mathrm{CR}$ is incremented by $1(\mathrm{CR}=7))$. As $\mathrm{CR}>$ Last Row, pass II is over.

Assign Label $=2$ to the corresponding marked cells in the Input Image (Figure 7). As FLAG is NOTPREVMARKED, LABEL is incremented by one. ( $\mathrm{LABEL}=3$ )

Pass III:

Get the first unmarked value from the PM (Figure 8a). The unmarked value is $10 . \mathrm{PV}[]=\{10\}$. Set FLAG $=$ NOTPREVMARKED.

Pass i: The adjacent values of 10 are 9, 10. AV []$=\{9,10\}$. Now $C R=1$. The AV values are searched in $C R$ and $\mathrm{CR}+1$ and those cells (10 in Row 1 and 10 in Row 2) are marked (Figure 8b).). CR is incremented by 1 $(\mathrm{CR}=2))$. The cell marked in CR is $10(\mathrm{PV}[]=\{10\}$.

Pass ii: The Adjacent values of 9 and 10 are $\operatorname{AV}[]=\{9,10\}$. Find those values in Row 1, 2 and 3 as $C R=2$ and those cells (10 in Row 3) are marked (Figure 8c). $\mathrm{CR}$ is incremented by $1(\mathrm{CR}=3))$. The cell marked in $\mathrm{CR}$ is $10(\mathrm{PV}[]=\{10\}$.

\begin{tabular}{|l|l|l|l|l|l|l|l|l|l|}
\hline 1 & 1 & 1 & 0 & 0 & 0 & 2 & 0 & 0 & 1 \\
\hline 0 & 1 & 1 & 0 & 0 & 2 & 0 & 2 & 0 & 1 \\
\hline 0 & 0 & 0 & 0 & 2 & 0 & 2 & 0 & 0 & 1 \\
\hline 0 & 0 & 0 & 2 & 0 & 2 & 0 & 2 & 2 & 0 \\
\hline 0 & 0 & 2 & 2 & 0 & 2 & 2 & 0 & 2 & 0 \\
\hline 2 & 2 & 0 & 0 & 2 & 2 & 0 & 2 & 0 & 2 \\
\hline 0 & 2 & 2 & 2 & 0 & 0 & 2 & 2 & 2 & 0 \\
\hline
\end{tabular}

Figure 7. Image (I) after Pass II.
Pass iii: The Adjacent values of 9 and 10 are AV []$=\{9,10\}$. Find those values in Row 2, 3 and 4 as $\mathrm{CR}=3$ and those cells (9 in Row 3) is already marked (Figure 8c). Assign LABEL $=2$ (LABEL of previously marked cell). $\mathrm{CR}$ is incremented by $1(\mathrm{CR}=4))$.

There is no cell marked in Row $3(\mathrm{AV}[]=\varnothing)$. Third Pass is Over as AV [] $=\varnothing$. Assign LABEL $=2$ to the corresponding marked cells in the Input Image (Figure 9). Increment LABEL by one. (LABEL $=3$ ).

No more unmarked cells are found. So, Iterations are over and Total_No_Components= 2 (LABEL-1). There are 2 components found and those components are labeled as 1 and 2 .

Two connected components labeled as 1 and 2 for the Input Image ( Figure 2a) is obtained as the output (Figure 9) after the algorithm is executed.

\section{Experimental Result}

The experimentation of the algorithm is implemented in Matlab Tool and was carried out on the ICDAR data set consisting of 250 different images and as well as some images were taken from the WEB. The images are converted in to binary image by applying otsu's threshold method $^{9}$. Some of the images taken for the experiment are shown in Figure 10. The experimental results of Figure 8 is shown in Table 1 .The experimental results in this research confirm that the new proposed method labels the connected component and gives the number of components present in the binary images with $100 \%$ accuracy.

\section{Conclusion and Future Work}

This paper projected a new positional connected component labeling algorithm to label the connected components and to give the number of components present in binary

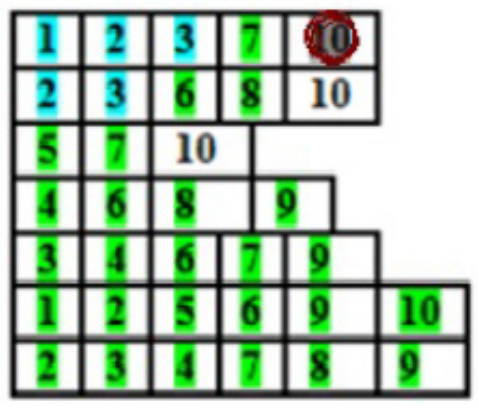

Figure 8a. First Unmarked Cell in Pass III.

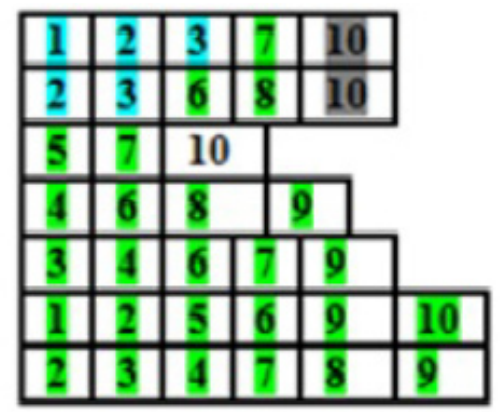

Figure 8b. Pass III-i.

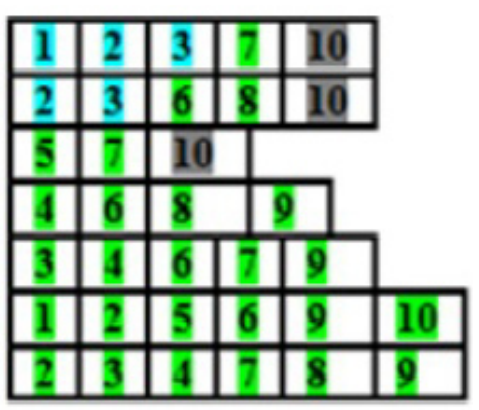

Figure 8c. Pass III Pass- ii. 


\begin{tabular}{|c|c|c|c|c|c|c|c|c|c|}
\hline & & & 0 & 0 & 0 & 2 & 0 & 0 & \\
\hline 0 & & & 0 & 0 & $\sqrt{2}$ & 0 & 2 & 0 & \\
\hline 0 & 0 & 0 & 0 & 2 & 0 & 5 & 0 & 0 & \\
\hline 0 & 0 & 0 & $\sqrt{2}$ & & 2 & 0 & 2 & 2 & \\
\hline 0 & 0 & 7 & 2 & 0 & 2 & 2 & 0 & 2 & \\
\hline$\sqrt{2}$ & 2 & 0 & 0 & 2 & $\sqrt{2}$ & 0 & 2 & 0 & \\
\hline 0 & 2 & $\sqrt{2}$ & $\sqrt{2}$ & $\overline{0}$ & 0 & $\sqrt{2}$ & & $\sqrt{2}$ & \\
\hline
\end{tabular}

Figure 9. Output Image (I) after Pass III.
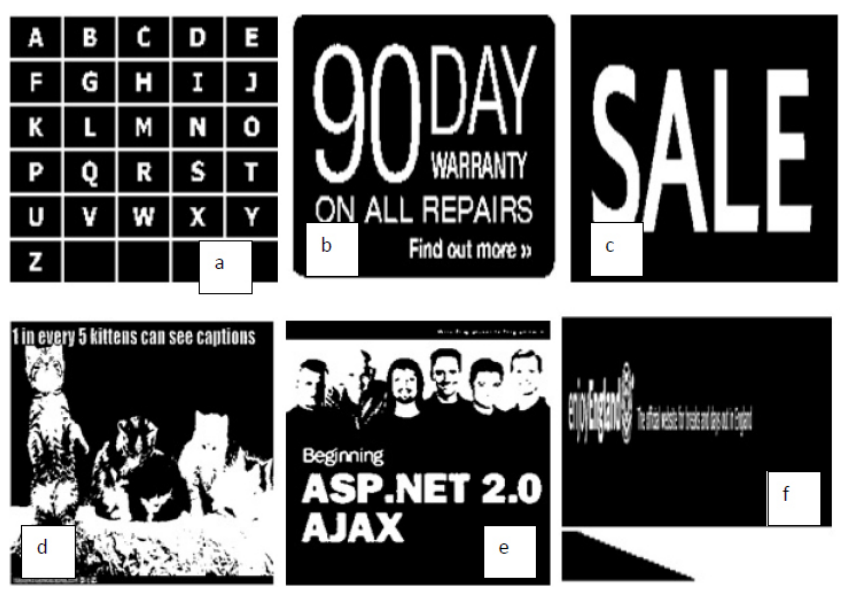

Figure 10. Sample Images (a) (b) (c) (d) (e) (f).

image. The proposed technique is an important stage for the object extraction method. Experiments are conducted by applying proper threshold technique on various images taken from ICDAR datasets and found that the outcome of the algorithm is $100 \%$. The future work focuses on developing a connected component labeling algorithm to label the connected component present in gray scale image.

\section{References}

1. Chang F, Chen C-J. A component-labeling algorithm using contour tracing technique. Proceedings of the Seventh
Table 1. Evaluated Number of Connected Component

\begin{tabular}{llc}
\hline Image & \multicolumn{1}{c}{ Size } & $\begin{array}{c}\text { No. of Connected } \\
\text { Component Found }\end{array}$ \\
\hline Fig 8a & $142 \times 178$ & 27 \\
Fig 8b & $125 \times 19$ & 36 \\
Fig 8c & $125 \times 300$ & 4 \\
Fig 8d & $359 \times 499$ & 220 \\
Fig 8e & $297 \times 275$ & 67 \\
Fig 8f & $126 \times 900$ & 57 \\
\hline
\end{tabular}

International Conference on Document Analysis and Recognition (ICDAR 2003). 2003 Aug 3-6. p.741-45.

2. Yuhai, L, Kuizhi, , Peixiang, D. An efficient and low memory requirement algorithm for extracting image component information, International Journal of Advanced Intelligence. 2011; 3(2):255-67.

3. Sutheebanjard P, Premchaiswadi W. Efficient scan mask techniques for connected components labeling algorithm, EURASIP Int J Image Video Process. 2011:14:1-20.

4. Niknam M, Thulasiraman P, Camorlinga S. A parallel algorithm for connected component labelling of gray-scale images on homogeneous multicore architectures. Journal of Physics: Conference Series. 2010; 256(1):1-8.

5. Ni Ma, Donald G. Bailey, Christopher T. Johnston. Optimised Single Pass Connected Components Analysis. FPT 2008. International Conference on ICECE Technology; 2008 Dec 8-10; Taipei; IEEE Proceedings p. 185-192.

6. Rakhmadi A, Rahim MSM, Bade A, Haron H, Amin IM. Loop back connected component labeling algorithm and its implementation in detecting face. Proc World Acad Sci Eng Tech. 2010; 40:83-88.

7. Bader DA, Joseph JáJá (1996). Parallel Algorithms for Image Histogramming and connected Components with an Experimental Study, J Parallel Distr Comput. 1996; 35:173-90.

8. Asano T, Tanaka H. In-place algorithm for connected components labeling., Journal of Pattern Recognition Research. 2010; 5(1):10-22.

9. Otsu N. A threshold selection method from gray-level histograms. IEEE Trans Syst Man Cybern. 1979; 9:62-6. 